\title{
Influence de la lumière et de la température sur les taux de croissance et de photosynthèse de Scenedesmus crassus Chodat, isolée de la retenue eutrophe El Kansera (Maroc)
}

\author{
M. Derraz 1 \\ A. Dauta 2 \\ J. Capblancq2 \\ M. Abassi1
}

Mots clés : Scenedesmus crassus, taux de croissance, activité photosynthétique, lumière, photopériode, température.

Les taux spécifiques de croissance et de photosynthèse en relation avec la température et la lumière, ont été déterminés pour Scenedesmus crassus, une algue isolée de la retenue eutrophe El Kansera. Les expériences ont été réalisées dans l'intervalle de température $\left[10-35^{\circ} \mathrm{C}\right]$ et pour des intensités lumineuses comprises entre 10 et $800{ }_{\mu} \mathrm{E} \cdot \mathrm{m}^{-2} \cdot \mathrm{s}^{-1}$, sous une photopériode de 15/9. Les résultats obtenus sont décrits par des fonctions mathématiques. Le taux spécifique de croissance maximal ( $\mu \max =1,72 \mathrm{j}^{-1}$ ) correspond à des conditions d'éclairement (Iopt) et de température (Topt) optimales respectivement égales à $312 \mu \mathrm{E} \cdot \mathrm{m}^{-2} \cdot \mathrm{s}^{-1}$ et $28^{\circ} \mathrm{C}$. Le taux de photosynthèse optimal (Pmax) et l'intensité seuil de saturation Ik(p) augmentent en fonction de la température. La valeur de Pmax la plus élevée $\left(4,40 \mathrm{mg} \mathrm{C}\right.$. $\left.\mathrm{mg} \mathrm{Chla}^{-1} \cdot \mathrm{h}^{-1}\right)$ est $^{-}$ obtenue à $35^{\circ} \mathrm{C}$ pour une valeur de $\mathrm{lk}(\mathrm{p})$ de $240{ }_{\mu} \mathrm{E} \cdot \mathrm{m}^{-2} \cdot \mathrm{s}^{-1}$. Une faible photoinhibition (moins de $5 \%$ ) de Pmax a été observée au-delà de $300 \mu \mathrm{E} \cdot \mathrm{m}^{-2} \cdot \mathrm{s}^{-1}$, alors que le taux maximum de croissance présente une nette diminution. L'analyse des résultats obtenus a fait apparaître une bonne corrélation de Pmax avec Ik(p) et $\mu$ max.

Growth and photosynthesis rates of Scenedesmus crassus Chodat, isolated from the eutrophic impoundment El Kansera (Morocco)

Keywords : Scenedesmus crassus, growth rate, photosynthetic activity, light, photoperiod, temperature.

For Scenedesmus crassus isolated from the eutrophic impoundment « El Kansera » (Morocco), the specific growth and photosynthesis rates were measured, over a range of light intensities $\left[10-800 \mu E \cdot \mathrm{m}^{-2} \cdot \mathrm{m}^{-1}\right]$ and temperatures from 10 to $35^{\circ} \mathrm{C}$, under $15 / 9$ light : dark cycle. Optimum conditions of irradiance (Iopt) and temperature (Topt) were $312 \mu \mathrm{E} \cdot \mathrm{m}^{-2} \cdot \mathrm{s}^{-1}$ and $28^{\circ} \mathrm{C}$, for the maximum specific growth $\left(\mu \max =1,72 \mathrm{j}^{-1}\right)$. Both photosynthetic rate $(P \max )$ and light saturation charactereristics $\mathrm{Ik}(\mathrm{p})$ increased with temperature. The high values of Pmax $\left(4,40 \mathrm{mgC}\right.$. $\left.\mathrm{mgChla}^{-1} \cdot \mathrm{h}^{-1}\right)$ and $\mathrm{Ik}(\mathrm{p})\left(240 \mu \mathrm{E} \cdot \mathrm{m}^{-2} \cdot \mathrm{s}^{-1}\right)$ were obtained at $35^{\circ} \mathrm{C}$. Little photoinhibition (less than $5 \%$ ) of Pmax was observed at irradiance exceeding $300 \mu \mathrm{E} \cdot \mathrm{m}^{-2} . \mathrm{s}^{-1}$, while the growth rate decreased markedly at irradiance above saturation. Pmax appears correlated with $\mathrm{Ik}(\mathrm{p})$ and $\mu \mathrm{max}$.

\section{Introduction}

En milieu naturel, le développement des espèces phytoplanctoniques et leur succession dépendent de l'interaction des facteurs qui contrôlent la croissance

\footnotetext{
1. Département de Biologie, Faculté des Sciences, B.P. 4010, Béni-M'hamed, Meknès, Maroc.

2. Laboratoire d'Hydrobiologie, Université Paul Sabatier, . URA CNRS 695, 118, route de Narbonne, 31062 Toulouse Cedex, France.
}

des populations (durée et intensité de l'éclairement, $\mathrm{pH}$, quantité de nutriments disponibles), avec ceux qui contrôlent leur disparition de la zone euphotique (respiration, sédimentation, broutage par le zooplancton) (Lin 1972, Okino 1973, Reynolds 1973, Reynolds \& Rogers 1976, Reynolds 1984, Coesel et al. 1994).

Une relation étroite entre la croissance et l'activité photosynthétique des algues a été montrée (Banse 1976, Harris 1978, Dauta 1982, Gibson 
\& Foy 1982, Post et al. 1985, Feuillade \& Feuillade 1987, Geider \& Osbonne 1992). De nombreux travaux ont décrit l'évolution de l'activité photosynthétique en fonction de la lumière par application de fonctions mathématiques, dont l'avantage est le calcul de paramètres caractéristiques, utiles à la comparaison des résultats (Smith 1936, Steele 1965, Jassby \& Plat 1976, Lederman \& Tett 1981, Peeters \& Eilers 1978, Eilers \& Peeters 1988, 1993).

L'objectif de la présente étude est de déterminer les caractéristiques de la photosynthèse et de la croissance d'une souche de Scenedesmus crassus abondante dans la zone littorale de la retenue eutrophe El Kansera (Maroc), sous l'influence des facteurs lumière et température. Le modèle dynamique de Eilers \& Peeters (1988), a été retenu pour ajuster les résultats expérimentaux.

\section{Matériel et méthodes}

La souche utilisée dans ce travail a été obtenue en 1992 à partir d'individus isolés dans des prélèvements provenant de la retenue du barrage El Kansera, en période estivale. Après une série de repiquages des colonies isolées dans des milieux de culture mélangés à l'eau de la retenue, nous avons obtenu une culture pure mais non axénique, et qui reste en développement sous sa forme coloniale.

La culture ainsi obtenue est maintenue confinée sur un milieu entièrement minéral (Dauta 1982) dont l'équilibre chimique autorise des cultures de longue durée. Afin de fournir un apport adéquat de dioxyde de carbone $\left(\mathrm{CO}_{2}\right)$, les cultures sont fortement bullées $\left(0,51\right.$ d'air. $^{\left.1^{-1}\right)}$ avec de l'air comprimé filtré (filtre Wathman GF/C de $1 \mu \mathrm{m}$ de porosité) insuflé à la base des flacons. Après stérilisation du milieu de culture à l'autoclave, le pH est ajusté à 7,8 par ajout de quelques gouttes de HCL dilué. Les expériences sont réalisées dans des enceintes thermorégulées, sous des conditions de température, de lumière et de photopériode définies (Boumnich et al. 1990).

La culture mère utilisée dans ces expériences est maintenue pure et en phase de croissance exponentielle par des repiquages fréquents (tous les 2 à 3 jours), sous des conditions de culture sub-optimales $\left(\mathrm{T}=25^{\circ} \mathrm{C}\right.$, IL $=$ $150 \mu$ E.m $\left.{ }^{-2} \cdot \mathrm{s}^{-1}\right)$.

\section{Mesure du taux de croissance}

Avant l'expérience destinée à la mesure du taux de croissance $\mu=\mathrm{f}(\mathrm{I}, \mathrm{T})$, la culture est placée pendant 24 heures à l'obscurité (synchronisation et non préadaptation à la lumière).
Sous une photopériode (lumière/obscurité) de $15 / 9$, et pour chaque température $\left(10,15,20,25,30\right.$ et $\left.35^{\circ} \mathrm{C}\right)$, la culture largement diluée dans un milieu neuf pour éviter tout effet d'autoombrage est répartie dans une série de flacons (Erlenmeyers de $300 \mathrm{ml}$ ), exposés à des intensités lumineuses comprises entre 10 et $800{ }_{\mu} \mathrm{E} \cdot \mathrm{m}^{-2} \cdot \mathrm{s}^{-1}$.

Le taux de croissance est déterminé à partir de la densité optique (mesuré à $750 \mathrm{~nm}$ dans une cuve de $5 \mathrm{~cm}$ ), représentative de la biomasse, avec une première mesure au début du cycle lumineux $\left(E \mathrm{t}_{0}\right)$, et après 24 heures d'incubation $\left(\mathrm{Et}_{1}\right)$. Le taux de croissance calculé correspond ainsi à la partie exponentielle de la courbe de croissance :

$$
\begin{aligned}
& \mu=\operatorname{Ln}\left(\mathrm{Et}_{1} / \mathrm{Et}_{0}\right) \\
& \text { pour un intervalle de } 1 \text { jour }
\end{aligned}
$$

\section{Mesure de l'activité photosynthétique}

Les cultures en phase exponentielle de croissance sont préconditionnées pendant 48 heures à de faibles intensités lumineuses $\left(10 \mu \mathrm{E} \cdot \mathrm{m}^{-2} \cdot \mathrm{s}^{-1}\right)$, et aux diverses températures où seront effectuées les mesures $\left(10\right.$ à $\left.35^{\circ} \mathrm{C}\right)$. La mesure de la photosynthèse est réalisée sur des cultures dans un milieu modifié par un ajout d'eau minérale (Vittel), très chargé en $\mathrm{HCO}_{3}-$, ce qui permet d'obtenir une alcalinité assez élevée ( $>2$ meq.1-1), évitant une diminution possible de la vitesse d'assimilation par une baisse trop rapide du substrat en carbone et des variations trop importantes de $\mathrm{pH}$ durant l'expérience.

Après un ajout de ${ }^{14} \mathrm{CO}_{3} \mathrm{NaH}$ (concentration finale de $10 \mu \mathrm{Ci} .^{-1}$ ), la culture est répartie dans des flacons de $60 \mathrm{ml}$ placés dans un dispositif conçu au laboratoire spécialement pour les expériences d'assimilation photosynthétiques de carbone. Ce dispositif (Fig. 1), réglable en température, permet d'incuber simultanément un grand nombre de flacons, répartis dans des valeurs d'intensité lumineuse comprises entre 10 et $800{ }_{\mu} \mathrm{E} \cdot \mathrm{m}^{-2} \cdot \mathrm{s}^{-1}$.

A la fin de l'incubation ( 40 à $60 \mathrm{mn}$ ), un volume de $15 \mathrm{ml}$ de chaque flacon est filtré sur membrane en nitrate de cellulose $(0,45 \mu \mathrm{m})$. Le filtre rincé avec de l'eau distillée ( 3 fois $5 \mathrm{ml}$ ) est transféré immédiatement dans des fioles contenant $5 \mathrm{ml}$ de liquide scintillant (Ready solv, $\mathrm{HP} / \mathrm{b}$, Beckmann) et la radioactivité du filtre est mesurée sur un compteur à scintillation Beckman LS $5000 \mathrm{CE}$. Un témoin (bruit de fond), pris à $t=0$ est déduit de la valeur obtenue.

Les concentrations en ${ }^{12} \mathrm{CO}_{2}$ ont été calculées pour chaque température à partir des valeurs de $\mathrm{pH}$ et d'alcalinité, et les teneurs en chlorophylle a ont été déterminées par la méthode spectrophotométrique décrite par Marker et al. (1980), en utilisant de l'ethanol $90 \%$ porté à ébullition pour l'extraction. Les résultats de Pmax sont exprimés en $\mathrm{mg} \mathrm{C}$ assimilé par $\mathrm{mg}$ de chlorophylle a et par heure (mgC. mg Chla-1.h' $\mathbf{h}^{-1}$ ). 


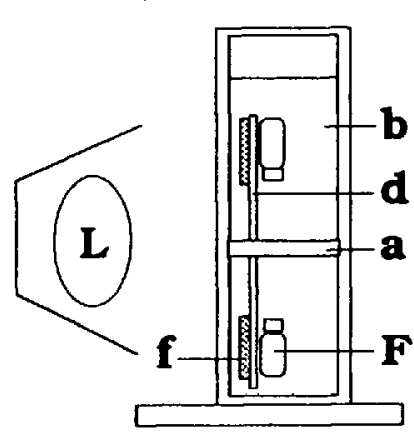

Profil

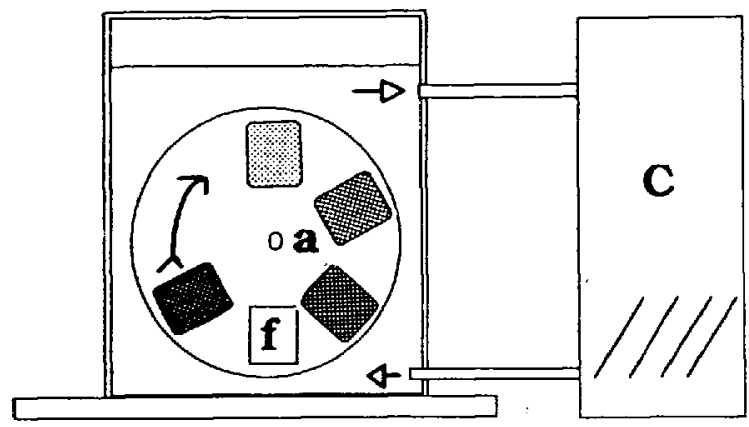

Face

Fig. 1. Représentation schématique du dispositif d'incubation pour mesure de la production primaire. $\mathrm{L}$ : lampe phytoclaude $400 \mathrm{w}-\mathrm{d}$ : disque rotatif - F : Flacon d'incubation - f : filtres de densité variable - $\mathrm{a}$ : axe de rotation du disque - $\mathrm{b}$ : bain d'eau thermostaté - $\mathrm{C}$ : cryostat.

Fig. 1. Schematic representation of the device used to measure the primary production.

L : phytoclaude $400 \mathrm{w}$ Lamp - $\mathrm{d}$ : rotating disk - F : incubation flask - $\mathrm{f}$ : light filter - a : axis of rotation - $\mathrm{b}$ : temperature regulated bath - $\mathrm{C}$ : cryostat.

La relation liant les taux de photosynthèse à l'intensité lumineuse est décrite par le modèle de Eilers \& Peeters (1988). Ce modèle dynamique a l'avantage de décrire les processus de photosynthèse, en relation avec la photoinhibition, par détermination des paramètres caractéristiques de la production : taux maximal de photosynthèse (Pmax), intensité lumineuse optimale (lopt) et influence de la photoinhibition (w) :

$$
\begin{gathered}
\mathrm{P} / \mathrm{Pmax}=\left((2+\mathrm{w})^{*} I^{\prime}\right) /\left(\mathrm{I}^{\prime 2}+\mathrm{w}^{*}\left(I^{\prime}+\mathrm{l}\right)\right) \\
\text { avec I' }=\mathrm{I} / \mathrm{Iopt} \\
\mathrm{w}=\text { coefficient de courbure de la fonction } \\
\text { (indicateur du potentiel photoinhibiteur) }
\end{gathered}
$$

D'autres paramètres utiles, l'intensité seuil ou initiale de saturation de la photosynthèse $l k(p)$ et le rendement de la production (pente initiale de la courbe : $\alpha(p)$ ) peuvent être tirés de l'équation de Eilers \& Peeters (1988) :

$$
\begin{gathered}
w=(\operatorname{lopt} / \operatorname{Ik}(p))-2 \\
\text { avec } \operatorname{Ik}(p)=\operatorname{Pmax} / \alpha(p)
\end{gathered}
$$

L'ajustement des valeurs expérimentales du modèle de Eilers \& Peeters (1988) en fonction de la température a été effectué selon la méthode de Lehman et al. (1975) :

$$
\begin{aligned}
& \operatorname{Pmax}(T)=\operatorname{Pmax}^{*} \mathrm{e}\left(-2,3^{*}(\mathrm{~T}-\mathrm{Topt})^{2} / \mathrm{b}^{2}\right) \\
& \text { avec } b=\text { Tsup - Topt si } \mathrm{T}>\text { Topt } \\
& =\text { Tmin }- \text { Topt } \quad \text { si } \mathrm{T}<\text { Topt }
\end{aligned}
$$

Les résultats de Pmax sont exprimés en mg C.mg Chla-1 $\cdot \mathrm{h}^{-1}$
Les données de la croissance de $S$. crassus en fonction de l'intensité lumineuse ont été analysées en applicant les mêmes modèles mathématiques. Le taux de croissance maximal $\mu$ max, l'intensité initiale de saturation $\operatorname{Ik}(\mathrm{g})$ et la pente initiale de croissance $\alpha(\mathrm{g})$, remplacent respectivement $\operatorname{Pmax}, \mathrm{Ik}(\mathrm{p})$ et $\alpha(\mathrm{p})$. La fonction (4) a été également utilisée dans l'ajustement de courbes des paramètres de croissance en fonction de la température.

\section{Résultats}

La figure 2 donne l'évolution du taux spécifique de croissance $(\mu)$ de $S$. crassus. Les valeurs des principaux paramètres associés à la croissance de cette espèce $\mu \max , \operatorname{Ik}(\mathrm{g}), \alpha(\mathrm{g})$ et w sont récapitulées dans le tableau I. Une relation linéaire entre le taux de croissance et la lumière n'apparaît qu'aux faibles intensités lumineuses ( $\mathrm{I}<100{ }_{\mu} \mathrm{E} \cdot \mathrm{m}^{-2} \cdot \mathrm{s}^{-1}$ ), et aux températures 10 et $15^{\circ} \mathrm{C}$; au-dessus de $100 \mu \mathrm{E} . \mathrm{m}^{-2} . \mathrm{s}^{-1}$ toute augmentation de l'intensité lumineuse reste sans effet sur la croissance de la culture de $S$. crassus à la photopériode 15/9.

Le taux de croissance maximal $(\mu \max =$ $\left.1,72 \mathrm{j}^{-1}\right)$, a été obtenu pour une intensité lumineuse de $312 \mu \mathrm{E} \cdot \mathrm{m}^{-2} \cdot \mathrm{s}^{-1}$ et une température de $28^{\circ} \mathrm{C}$. Le degré d'inhibition de la croissance, déterminé par calcul de $w$, commence à des niveaux d'intensité lumineuse variables avec la température. Les effets 
Taux de croissance $\left(\mathrm{j}^{-1}\right)$
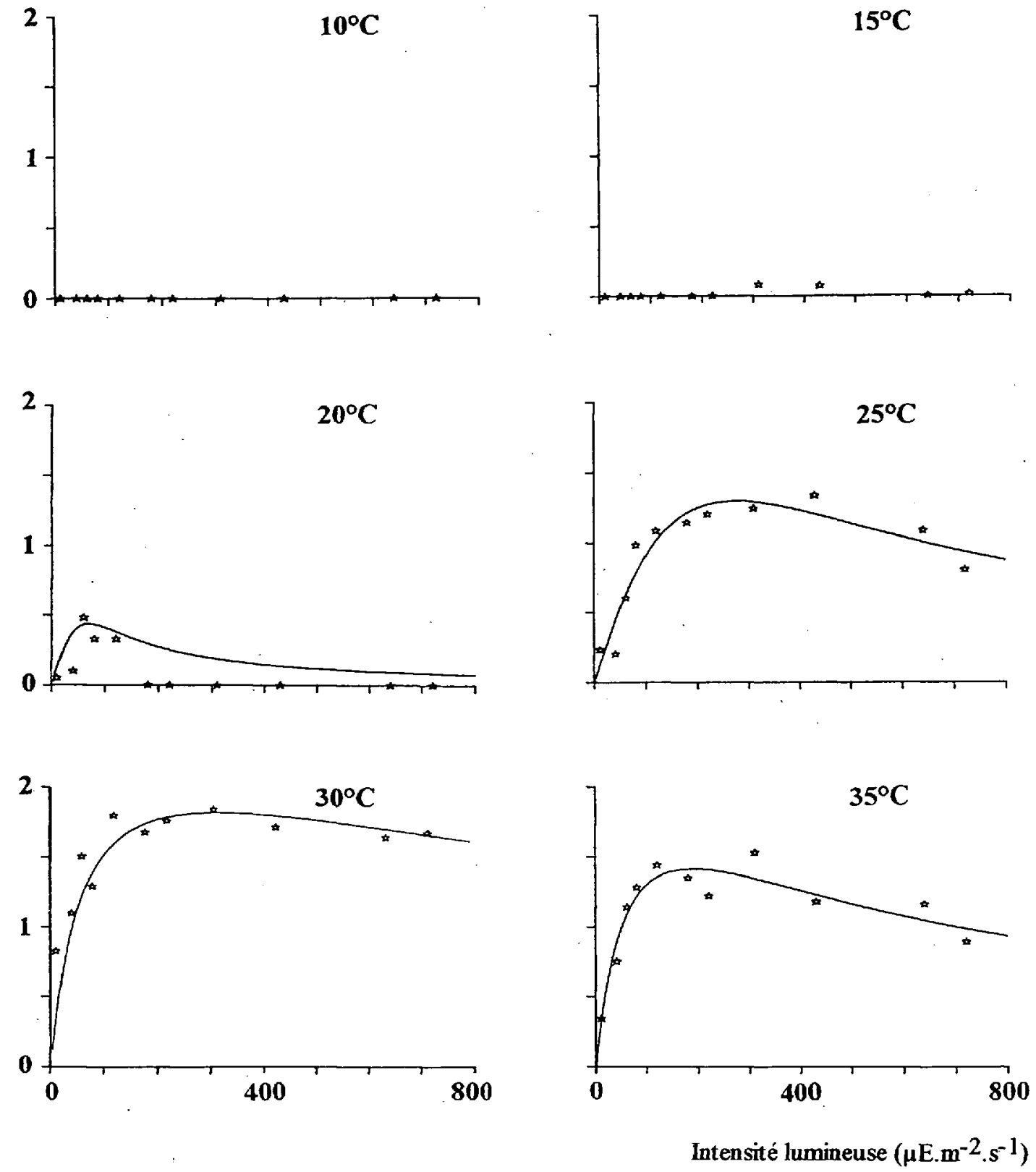

Fig. 2. Taux de croissance de Scenedesmus crassus, en relation avec la lumière et la température (ajustement par le modèle de Eilers \& Peeters 1988).

Fig. 2. Growth rate of Scenedesmus crassus in relation to irradiance and temperature. (Curves adjustement by Eilers \& Peeters 1988). 
interdépendants de la lumière et de la température sur le taux de croissance se traduisent par l'évolution de ${ }_{\mu} \max$ et Iopt en fonction de la température (Fig. 4).

L'intensité seuil de saturation de la croissance Ik(g) montre peu de variation, sauf à la température $20^{\circ} \mathrm{C}$ où nous avons obtenu une valeur relativement faible.

La capacité photosynthétique suit le même modèle (Fig. 3). Le taux maximal de photosynthèse (Pmax) et l'intensité seuil de saturation $\operatorname{Ik}(p)$ augmentent avec la température et atteignent $4,40 \mathrm{mg}$ C.mg chla-1 $^{-1}$ h-1 $^{-1}$ et $240 \mu \mathrm{E} \cdot \mathrm{m}^{-2} \cdot \mathrm{s}^{-1}$ respectivement (Fig. 5). Cependant le rendement de l'utilisation de la lumière pour la photosynthèse $\alpha(p)$ montre peu de variation (Tableau 2).

La photoinhibition de la photosynthèse est relativement plus faible que celle de la croissance.
Le degré d'inhibition le plus élevé (moins de $5 \%$ de Pmax) a été observé au-delà de $300 \mu \mathrm{E} \cdot \mathrm{m}^{-2} . \mathrm{s}^{-1}$ et à $30^{\circ} \mathrm{C}$.

L'analyse de ces résultats fait apparaître l'existence de corrélations significatives entre Pmax et Ik(p) d'une part (Fig. 6), et entre Pmax et $\mu$ max d'autre part (Fig. 7). Cette relation semble indiquer que le rapport Respiration / Pmax est relativement constant quelles que soient la température et l'intensité de la lumière.

\section{Discussion}

Les paramètres de croissance et de photosynthèse de Scenedesmus crassus, isolée d'un lac de barrage au Maroc, constituent une source de nouvelles données utiles à la compréhension des capacités d'adaptation de cette espèce aux différentes conditions environnementales.

Tableau 1. Paramètres de la croissance de Scenedesmus crassus aux différentes températures expérimentales.

Table 1. Growth characteristics of Scenedesmus crassus at different temperatures.

\begin{tabular}{|c|c|c|c|c|c|c|}
\hline température $\left({ }^{\circ} \mathrm{C}\right)$ & 10 & 15 & 20 & 25 & 30 & 35 \\
\hline $\begin{array}{l}\operatorname{\mu max} \\
\text { jour }^{-1} \text { ) }\end{array}$ & - & - & 0,44 & 1,30 & 1,72 & 1,41 \\
\hline$\underset{\left(\mu \mathrm{E} \cdot \mathrm{m}^{-2} \cdot \mathrm{s}^{-1}\right)}{\mathbf{I}_{\mathbf{k}}(\mathbf{g})}$ & - & - & 62 & 119 & 96 & 86 \\
\hline $\begin{array}{l}\alpha_{(\mathbf{g})} \\
\left(\mathrm{mgC} \cdot \mathrm{mg} \mathrm{chla}^{-1} \cdot \mathrm{h}^{-1}\right) \cdot\left(\mu \mathrm{E} \cdot \mathrm{m}^{-2} \cdot \mathrm{s}^{-1}\right)^{-1}\end{array}$ & - & - & 0,007 & 0,011 & 0,018 & 0,016 \\
\hline $\mathbf{w}$ & - & - & $-0,87$ & 0,29 & 1,25 & 0,18 \\
\hline
\end{tabular}

Tableau 2. Paramètres de la photosynthèse de Scenedesmus crassus aux différentes températures expérimentales. Table 2. Photosynthetic characteristics of Scenedesmus crassus at different temperatures.

\begin{tabular}{|c|c|c|c|c|c|c|}
\hline température $\left({ }^{\circ} \mathrm{C}\right)$ & 10 & 15 & 20 & 25 & 30 & 35 \\
\hline $\begin{array}{l}\text { Pmax } \\
\left(\operatorname{mgC} \cdot \operatorname{mg~chla} a^{-1} \cdot h^{-1}\right)\end{array}$ & 1,06 & 2,57 & 2,83 & 3,77 & 4,04 & 4,40 \\
\hline$\underset{\left(\mu \mathrm{E} \cdot \mathrm{m}^{-2} \cdot \mathrm{s}^{-1}\right)}{\mathbf{I}}$ & 47. & 68 & 142 & 188 & 230 & 240 \\
\hline $\begin{array}{l}\alpha_{(\mathbf{p})} \\
\left(\mathrm{mgC} \cdot \mathrm{mg} \mathrm{chla}^{-1} \cdot \mathrm{h}^{-1}\right) \cdot\left(\mu \mathrm{E} \cdot \mathrm{m}^{-2} \cdot \mathrm{s}^{-1}\right)^{-1}\end{array}$ & 0,022 & 0,037 & 0,019 & 0,020 & 0,017 & 0,018 \\
\hline w & 3 & 2,23 & 0,06 & 0,08 & $-0,62$ & $-0,28$ \\
\hline
\end{tabular}


Photosynthèse (mgC (mgchla $\left.)^{-1} \cdot h^{-1}\right)$
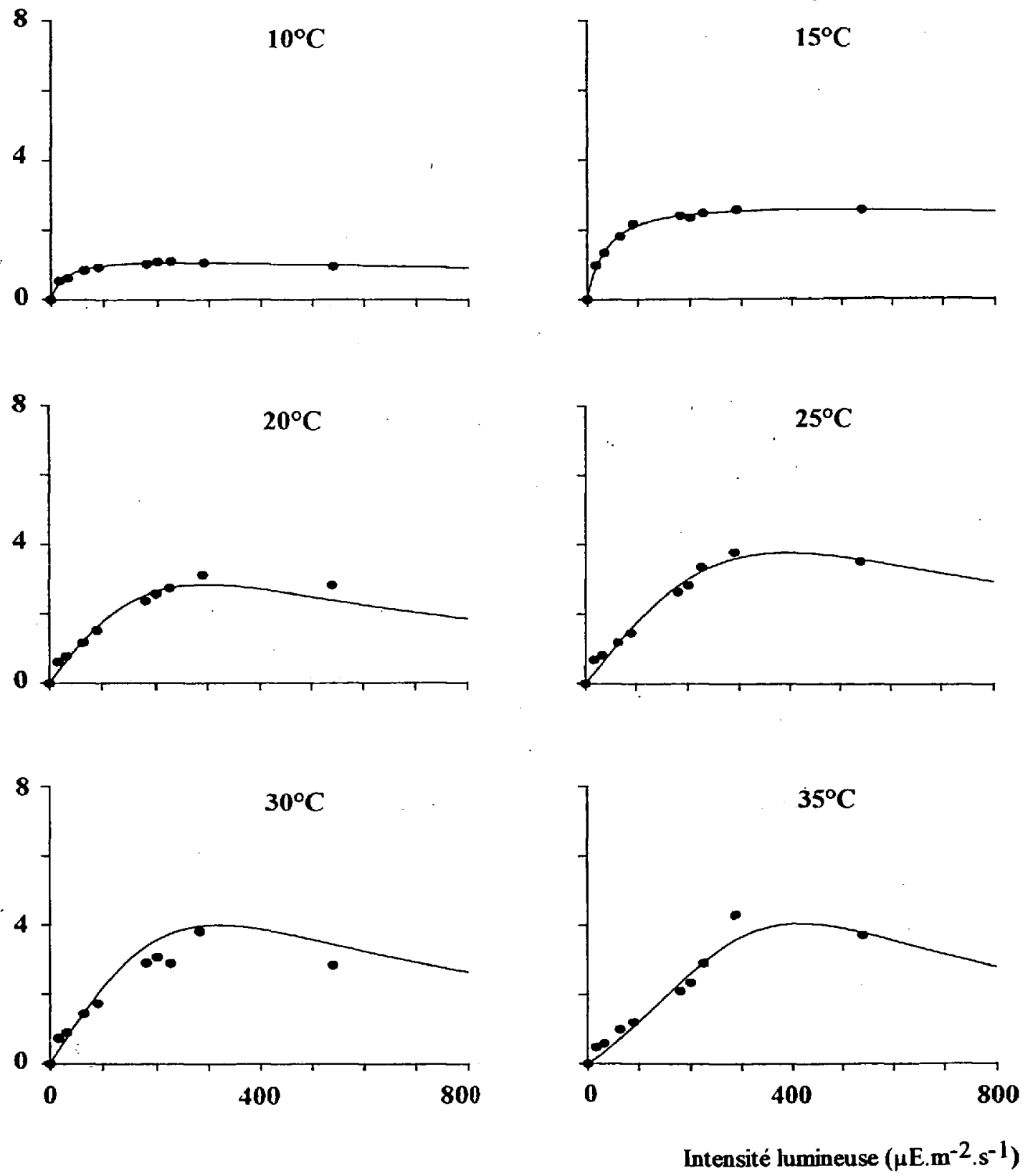

Fig. 3. Taux de photosynthèse de Scenedesmus crassis, en relation avec la lumière et la température (ajustement par le modèle de Eilers \& Peeters 1988).

Fig. 3. Photosynthetic rate of Scenedesmus crassus in relation to irradiance and temperature. (Curves adjustement by Eilers \& Peeters 1988). 

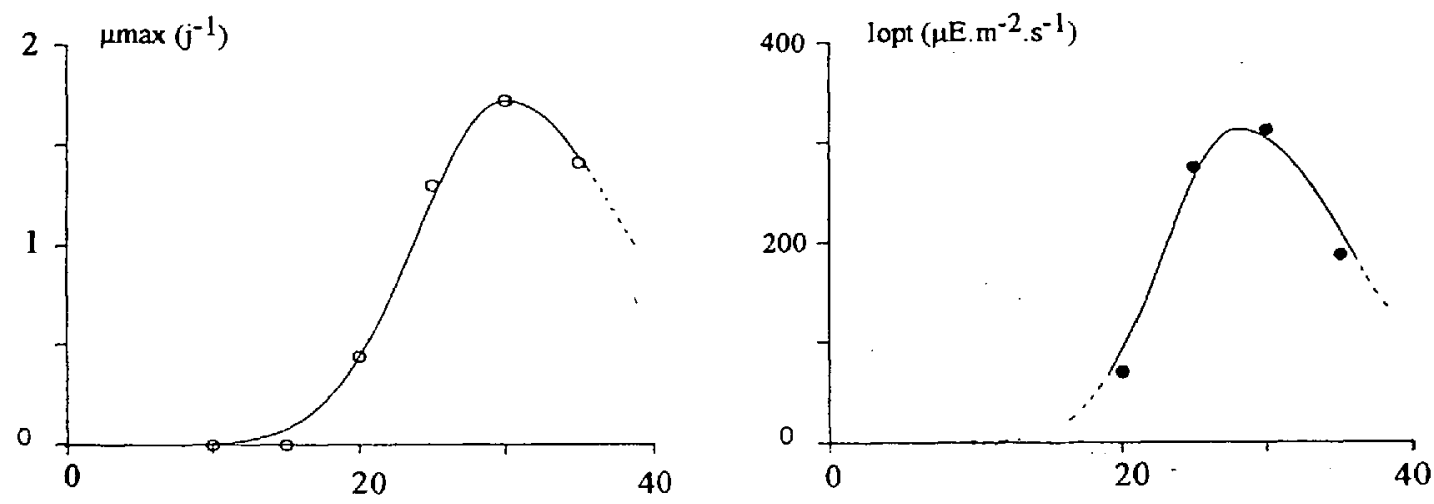

Température $\left({ }^{\circ} \mathrm{C}\right)$

Fig. 4. Variations des paramètres de croissance $\mu$ max et Iopt de Scenedesmus crassus en fonction de la température.

Fig. 4. Growth constants $\mu \max$ and Iopt for Scenedesmus crassus, as function of temperature.
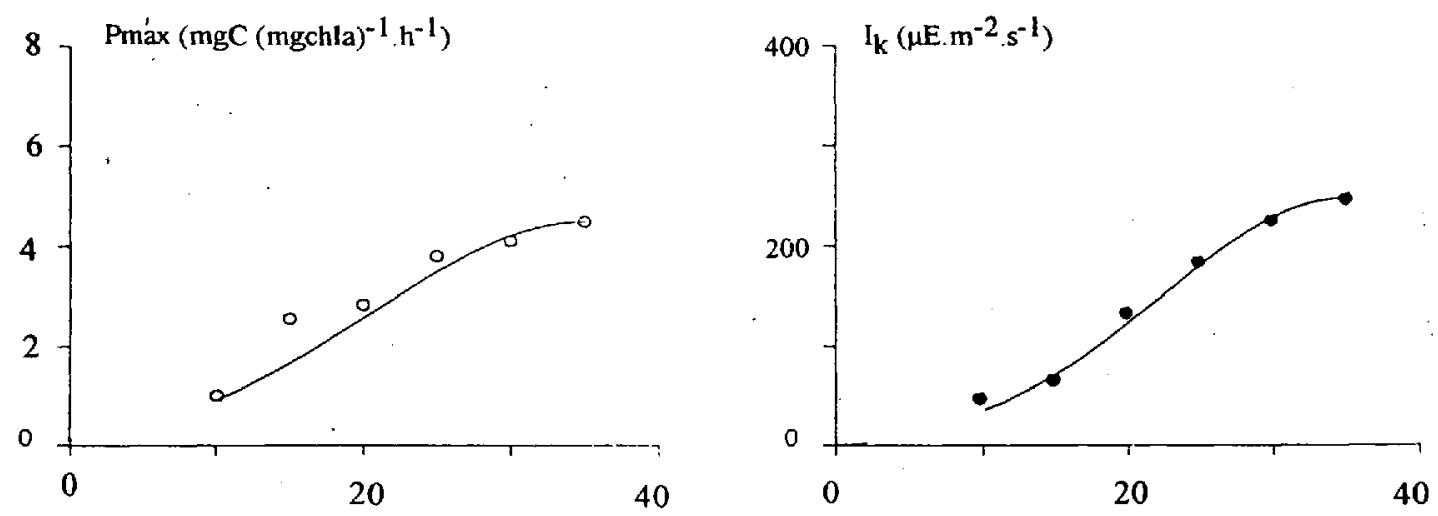

\section{Température $\left({ }^{\circ} \mathrm{C}\right)$}

Fig. 5. Effets de la température sur les paramètres de la photosynthèse Pmax et Ik(p) de Scenedesmus crassus.

Fig. 5. Effects of temperature on photosynthetic constants Pmax and Ik(p) for Scenedesmus crassus.

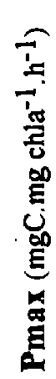

T1

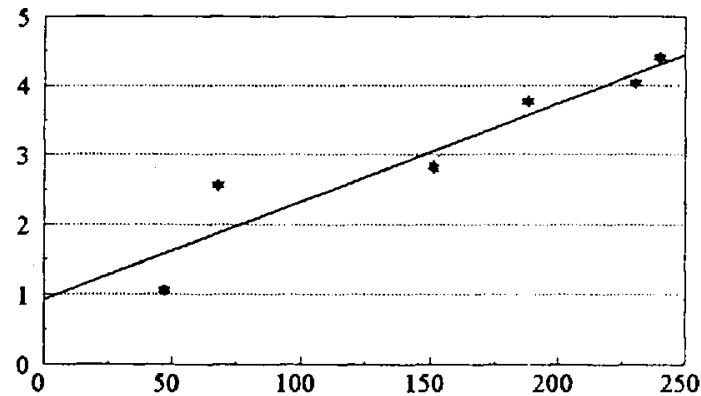

$$
\mathbf{l}(\mathbf{p})\left(\mu \mathrm{E} \cdot \mathrm{m}^{-2} \cdot \mathrm{s}^{-1}\right)
$$

Fig. 6. Variation de Pmax en fonction de Ik(p) pour S. crassus. Fig. 6. Relationship between characteristic parameters Pmax and $\mathrm{Ik}(p)$ for $S$. crassus.

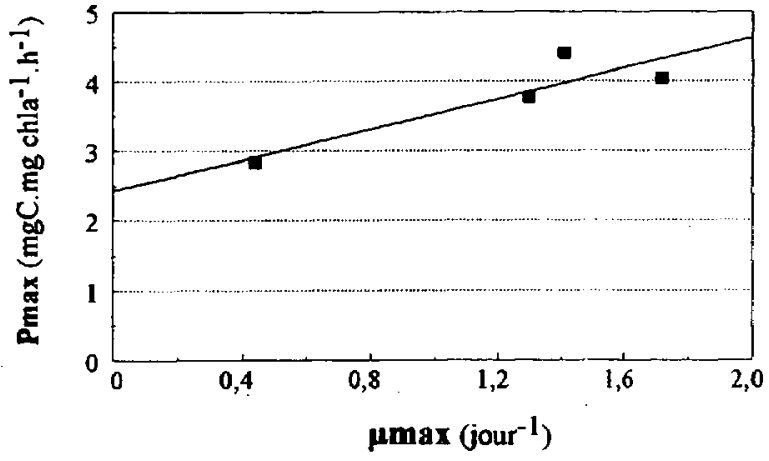

Fig. 7. Variation de Pmax en fonction de $\mu \max$ pour S. crassus. Fig. 7. Relationship between characteristic parameters Pmax and $\mu \max$ for S. crassus. 
La valeur du taux spécifique de croissance maximale la plus élevée $\left(1,72 \mathrm{j}^{-1}\right)$ est comprise dans l'intervalle des taux $\left[1-1,75 \mathrm{j}^{-1}\right]$ associés aux groupes des Chlorococcales (Schnoor \& Di Toro 1980). Cette valeur est proche de celle obtenue pour $S$. quadricauda $1,79 \mathrm{j}^{-1}$ ) par Oh \& Rhee (1991), mais elle est un peu plus élevée que la valeur trouvée par Ahlgreen (1987) pour une autre souche de $S$. quadricauda $\left(1,06 \mathrm{j}^{-1}\right)$, et ce à des températures de 20 à $25^{\circ} \mathrm{C}$ respectivement. Ces variations, mises en évidence pour des cultures non limitées en nutriments, reflèteraient l'influence de nombreux processus physiologiques et métaboliques (Reynolds 1984).

En plus de l'influence de la lumière, la croissance des algues est également sous la dépendance de la température (Goldman \& Carpenter 1974, Goldman 1977, Ahlgreen 1987). Cette dépendance a été bien démontrée dans notre cas par l'allure parabolique de l'évolution de $\mu$ max entre les températures 20 et $30^{\circ} \mathrm{C}$ (Fig. 4). Rhee \& Gotham (1981) et Ahlgreen (1987) ont obtenu des résultats similaires pour d'autres espèces de Scenedesmus, mais à des intervalles plus bas $\left[10-20^{\circ} \mathrm{C}\right]$ et $\left[3-25^{\circ} \mathrm{C}\right]$ respectivement.

Le taux de croissance maximal ( $\mu$ max) et l'intensité lumineuse optimale (lopt) correspondante, évoluent de la même manière avec la température. L'importante variation de Iopt est attribuée en particulier au pouvoir d'adaptation de certaines espèces d'algues aux diverses intensités de la lumière (Jorgensen 1969, Raps et al. 1983).

En ce qui concerne l'étude des paramètres de photosynthèse, nos résultats (Fig. 6 et 7) confirment, d'une part, la bonne corrélation entre la capacité photosynthétique optimale (Pmax) et l'intensité seuil de saturation de la photosynthèse $\mathrm{Ik}(\mathrm{p})$ (Dokulil 1984, Ahlgreen 1987) et, d'autre part, ils confirment également l'existence d'une assez bonne correspondance entre Pmax et $\mu \max$ (Ahlgreen 1987, Oh \& Rhee 1991). Les valeurs de Pmax obtenues pour $S$. crassus varient de 1,06 à 4,40 mgC.mg chla-1.h-1 dans l'intervalle $\left[10-35^{\circ} \mathrm{C}\right]$. Ces valeurs restent proches de celles trouvées par Ahlgreen (1987) et celles reportées par Oh \& Rhee (1991) pour $S$. quadricauda.

Selon Coesel et al. (1994), la faible photoinhibition obtenue dans le cas de la photosynthèse est en relation avec la diminution des teneurs cellulaires en chlorophylle a. De même, Oh \& Rhee (1991) ont établi une relation inversement proportionnelle entre
Pmax et le rapport chlorophylle a : carbone. Nos résultats suggèrent que les fortes lumières inhibent davantage la croissance que la photosynthèse. Ceci s'explique par les conditions d'éclairement différentes auxquelles les algues sont soumises lors des mesures de croissance et de photosynthèse (Belay \& Fogg 1978, Harris 1978, Foy 1983). Les mesures de photosynthèse nécessitent en effet un temps d'exposition à la lumière beaucoup plus court que les mesures de croissance, ces dernières étant réalisées, par ailleurs, sur des cultures préalablement conditionnées à l'obscurité.

La technique de mesure de l'activité photosynthétique et les conditions de conversion utilisées dans la littérature (relation chlorophylle a : carbone cellulaire, oxygène ou densité cellulaire) rendent le plus souvent ces comparaisons assez difficiles, et sont en partie responsables des variations de Pmax. Dans ces conditions, les paramètres $\alpha(p)$ et Ik $(p)$ sont généralement utilisés avec le paramètre Pmax pour définir la relation qui lie la photosynthèse à la lumière, surtout $\mathrm{Ik}(\mathrm{p})$ qui a l'avantage d'être indépendante des unités utilisées dans l'expression de la capacité photosynthétique (Henley 1993).

L'intensité seuil de saturation de la photosynthèse $\mathrm{Ik}(\mathrm{p})$, évolue dans le même sens que la température. Elle varie dans une plage assez étroite : entre 47 et $240 \mu \mathrm{E} \cdot \mathrm{m}^{-2} . \mathrm{s}^{-1}$, alors que le rendement de la lumière pour la photosynthèse $\alpha(p)$ ne montre pas de variations significatives. De nombreux travaux (Raven \& Geider 1988, Davison 1991, Henley 1993) ont montré que $\alpha(\mathrm{p})$ est moins affecté par la température que $I k(p)$ et Pmax.

La comparaison entre les valeurs de Ik pour la croissance $\mathrm{Ik}(\mathrm{g})$ et la photosynthèse $\mathrm{Ik}(\mathrm{p})$ a montré que l'intensité seuil de saturation est significativement plus élevée pour la photosynthèse que pour la croissance. Cette différence a été largement citée dans la littérature pour beaucoup d'autres espèces (Fogg 1983, Richardson et al. 1983, Zevenboom \& Mur 1984, Raps et al. 1983, Oh \& Rhee 1991, Nielsen 1992) ; elle démontre que le carbone assimilé par photosynthèse n'est pas incorporé entièrement pour la croissance (Fogg 1983). Quoique la conversion des taux de photosynthèse en taux de croissance suppose la prise en considération de paramètres tels que le quota cellulaire en carbone et la respiration, une bonne correspondance entre Pmax et $\mu \max$ de $S$. crassus a été observẹe (Fig. 7). 
Les travaux de Oh \& Rhee (1991), effectués sur plusieurs espèces isolées d'un même milieu ont précisé que le rendement du carbone fixé par photosynthèse pour la croissance, déterminé par le rapport taux spécifique de croissance : capacité photosynthétique $(\mu / \mathrm{P})$, peut constituer une caractéristique spécifique importante dans la succession écologique des communautés phytoplanctoniques.

\section{Remerciements}

Cette étude a été réalisée au Laboratoire d'Hydrobiologie de Toulouse, URA CNRS 695, dans le cadre de stages financés par le CNRS et l'AUPELF (Association des Universités Partiellement ou Entièrement de Langue Française).

\section{Travaux cites}

Ahlgreen G. 1987. - Temperature functions in biology and their application to algal growth constants. Oikos, $49: 177-190$.

Banse K. 1976. - Rates of growth, respiration and photosynthesis of unicellular algae as related to cell size- a review. $J$. Phycol., 12 : 135-140.

Belay A. \& Fogg G.E. 1978. - Photoinhibition of photosynthesis in Asterionella formasa (Bacillariophyceae). J. Phycol., $14: 341-347$.

Boumnich L., Dauta A., Devaux J. \& Romagoux J.C. 1990. Influence de la lumière et de la température sur la croissance de quatre espèces d'algues d'un lac eutrophe (Lac d'Aydat, Puy de Dôme, France). Annls Limnol., 26 : 3-10.

Capblancq J. 1982 . - Phytoplancton et production primaire. In Ecologie du plancton des eaux continentales. Pourriot $R$. (Ed.), Masson, Paris, $1-48$.

Coesel P.F.M. \& Wardenaar K. 1994. - Light-limited growth and photosynthetic characteristics of two planktonic desmid species. Freshwater Biology, 31 : 221-226.

Dauta A. 1982. - Conditions de développement du phytoplancton. Etude comparative du comportement de huit espèces en culture. 1. Détermination des paramètres de croissance en fonction de la lumière et de la température. Annls Limnol., $18: 217-262$.

Davison I.R. 1991. - Environmental effects on algal photosynthesis : temperature. J. Phycol., $27: 2-8$.

Dokulil M. 1984. - Assessment of components controlling phytoplankton photosynthesis and bacterioplankton production in a shallow, alkaline, turbid lake (Neusiedlersee, Austria). Int. Rev. ges. Hydrobiol., 69 : 679-727.

Eilers P.H.C. \& Peeters J.C.H. 1988, - A model for the relationship between light intensity and the rate of photosynthesis in phytoplankton. Ecol. Model., 2 : 199-215.

Eilers P.H.C. \& Peeters J.C.H. 1993. - Dynamic behaviour of a model for photosynthesis and photoinhibition. Ecol. Model., 69 : 113-133.

Feuillade J.B. \& Feuillade M. 1987. - Modelling steady-state growth and photosynthesis rates of Oscillatoria rubescens continuous cultures in relation to temperature and irradiance. J. Plankton Res., 9 : 445-157.

Fogg G.E. 1983. - The ecological significance of extracellular products of phytoplankton photosynthesis. Bot. mar., 26 : 3-14.
Geider R.J. \& Osborne B.A. 1992. - Algal photosynthesis. The measurement of algal gas exchange. Chapman \& Hall, New York, London : $256 \mathrm{p}$.

Gibson C.E. \& Foy R.H. 1983. - The photosynthesis and growth efficiency of a planktonic blue-green algae, Oscillatoria redekei. Br. Phycol. J., 18 : 39-45.

Goldman J.C. \& Carpenter E.J. 1974. - A kinetic approach to the effect temperature on algal growth. Limnol. Oceanogr., $19: 756-765$.

Goldman J.C. 1977. - Temperature effects on phytoplankton growth continuous culture. Limnol, Oceanogr., 22 : 932-936.

Harris G.P. 1978. - Photosynthesis, production and growth : The physiological ecology of phytoplankton. Arch. Hydrobiol. Beih. Ergebn. Limnol., 10-171.

Henley W.J. 1993. - Measurement and interpretation of photosynthetic light-response curves in algae in the context of photoinhibition and diel changes. J. Phycol., 29 : 729-739.

Jassby A.D. \& Platt T. 1976. - Mathematical formulation of the relationship between photosynthesis and light for phytoplankton. Limnol. Oceanogr., 21 : 540-547.

Jewsen D.H. 1976. - The interaction of components controling net phytoplankton photosynthesis in a well-mixed lake (Lough Neagh, Northern Ireland). Fresh water Biology, 6 : 551-576.

Jorgensen E.G. 1969. - The adaptation of plankton algae. IV. Light adaptation in different algal species. Physiol. plant., $22: 1307-1315$.

Lederman T.C. \& Tett P. 1981. - Problems in modelling in photosynthesis- light relationship for phytoplankton. Bot. Mar., 24 : 125-134.

Lehman J.T., Botkin D.B. \& Likens G.E. 1975. - The assumptions and rationales of a computer model of phytoplankton population dynamics. Limnol. Oceanogr., $20: 343-364$.

Lin C.K. 1972. - Phytoplankton succession in a eutrophic lake with special reference to the blue-green algal blooms. Hydrobiologica, $39:$ 321-334.

Marker A.F.H., Nush E.A. \& Riemann B. 1980. - The measurements of photosynthetic pigments in freshwater and standardization of methods : conclusions and considerations. Ergebn. Limnol., 14 : 91-106.

Nielsen M.V. 1992. - Irradiance and daylength effects on growth and chemical composition of Gyrodinium aureolum Hulburt in culture. J. Plankton Res., 14 : 811-820.

Oh H.M. \& Rhee G.Y. 1991. - A comparative study microalgae isolated from flooded rice paddlies : light-limited growth, $C$ fixation, growth efficiency and relative $\mathrm{N}$ and $\mathrm{P}$ requirement. J. of Applied Phycol., 3 : 211-220.

Okino T. 1973. - Studies on the blooming of Microcystis aeruginosa. Jap. J. Botany, $20: 381-402$.

Peeters J.C. \& Eilers P. 1978. - The relationship between light intensity and photosynthesis : a simple mathematical model Hydrobiol. Bull., 1 : 134-136.

Post A.F., De Witt R. \& Mur L.R. 1985. - Interactions between temperature and light intensity on growth and photosynthesis of Cyanobacterium Oscillatoria agardhii. J. Plankton Res., $7: 487-495$.

Raps S., Wyman K., Siegelman H.W. \& Falkowski P.G. 1983. - Adaptation of the Cyanobacterium Microcystis aeruginosa to light intensity. Plant Physiol., $72: 829-832$.

Raven J.A. \& Geider R.J. 1988. - Temperature and algal growth. New Phytol., 93 : 157-193. 
Reynolds C.S. 1973. - Growth and buoyancy of Microcystis aeruginosa Kütz Emend. Elenkin in shallow eutrophic lake. Proc. $R$. Soc. Lond., 184 : 29-50.

Reynolds C.S. \& Rogers D.A. 1976. - Seasonal variations in the vertical distribution and buoyancy of Microcystis aeruginosa Kütz Emend. Elenkin in Rostheme mere. EnglandHydrobiol., 48 : 17-23.

Reynolds C.S. 1984. - The ecology of freshwater phytoplankton. Cambridge University Press, London : $384 \mathrm{p}$.

Rhee G.Y. \& Gothman I.J. 1981. - The effect of environmental factors on phytoplankton growth : temperature and the indicators of temperature with nutrient limitation. Limnol. Oceanogr., $26:$ 635-648.
Richardson K., Beardall J. \& Raven J.A. 1983. - Adaptation of unicellular algae to irradiance : an analysis of strategies. New Phytol., 93 : 157-191.

Schnoor J.L. \& Di Toro D. 1980. - Differential phytoplankton sinking and growth rates: An eigenvalue Analysis. Ecol. Modelling., 9 : 233-245.

Smith E.L. 1936. - Photosynthesis in relation to light and carbon dioxid. Proc. Nat. Acad. Sc. Wash., 22 : 504-511.

Steele J.H. 1965. - Notes on some theoritical problems in production ecology. Mem. Ist. Ital. Idrobiol., 18 suppl, : 383-398.

Zevenboom W. \& Mur L.R. 1984. - Growth and photosynthesis response of the Cyanobacterium Microcystis aeruginosa in relation to photoperiodicity and irradiance. Arch. Microbiol., $139: 232-239$. 